\title{
Computational optical biopsy
} Yi Li*1, Ming Jiang ${ }^{2}$ and Ge Wang1,2

Address: ${ }^{1}$ Department of Mathematics, University of Iowa, Iowa City, IA 52242, USA and ${ }^{2}$ Bioluminescence Tomography Laboratory, Departments of Radiology and Biomedical Engineering, University of Iowa, Iowa City, IA 52242, USA

Email: Yi Li* - yi-li@uiowa.edu; Ming Jiang - ming-jiang@ieee.org; Ge Wang - ge-wang@ieee.org

* Corresponding author

Published: 14 June 2005

BioMedical Engineering OnLine 2005, 4:36 doi:10.1/86/1475-925X-4-36
Received: 0I April 2005

Accepted: 14 June 2005

This article is available from: http://www.biomedical-engineering-online.com/content/4/I/36

(c) 2005 Li et al; licensee BioMed Central Ltd.

This is an Open Access article distributed under the terms of the Creative Commons Attribution License (http://creativecommons.org/licenses/by/2.0), which permits unrestricted use, distribution, and reproduction in any medium, provided the original work is properly cited.

\begin{abstract}
Optical molecular imaging is based on fluorescence or bioluminescence, and hindered by photon scattering in the tissue, especially in patient studies. Here we propose a computational optical biopsy (COB) approach to localize and quantify a light source deep inside a subject. In contrast to existing optical biopsy techniques, our scheme is to collect optical signals directly from a region of interest along one or multiple biopsy paths in a subject, and then compute features of an underlying light source distribution. In this paper, we formulate this inverse problem in the framework of diffusion approximation, demonstrate the solution uniqueness properties in two representative configurations, and obtain analytic solutions for reconstruction of both optical properties and source parameters.
\end{abstract}

\section{Introduction}

Gene therapy is a breakthrough in the modern medicine, which promises to cure diseases by modifying gene expression. A key for development of gene therapy is to monitor the in vivo gene transfer and its efficacy in the mouse model. Traditional biopsy methods are invasive, insensitive, inaccurate, inefficient, and limited in the extent. To map the distribution of the administered gene, reporter genes such as those producing luciferase are being used to generate light signals within a living mouse, which can be externally measured [1]. A cooled highly sensitive CCD camera has been built to take a 2D view of expression of the bioluminescent reporter luciferases. Such a 2D image of photon emission is then superimposed onto a $2 \mathrm{D}$ visible light picture of the mouse for localization of the reporter gene activity. In addition to gene therapy, this new imaging tool has great potentials in various other biomedical applications as well. An in vivo bioluminescence tomography system integrated with an $\mathrm{X}$-ray CT/micro-CT scanner is recently reported in $[2,3]$. The novel concept is to collect emitted photons from multiple 3D directions with respect to a living mouse marked by bioluminescent reporter luciferases, and reconstruct an internal bioluminescent source distribution based on both the outgoing bioluminescent signals and the CT/microCT volume of the mouse. Then, the $3 \mathrm{D}$ bioluminescent source distribution and the corresponding CT/micro-CT volume are registered of anatomical and pathological structures, such as the lung and various tumors.

Optical imaging of small animals based on fluorescent/ bioluminescent probes promises great opportunities for translational research and eventually clinical applications, because fluorescent/ bioluminescent signals directly reveal molecular and cellular activities, and are sensitive, specific, non-ionizing, non-invasive and cost-effective. 
Pure optical imaging cannot detect the molecular activities triggered by biomarkers because the light generated are generally out of the visible spectrum. Despite the progress in optical molecular imaging of small animals, little research has been done for optical molecular imaging of patients. A light source induced by either fluorescence or bioluminescence probes is usually weak, and would be often deep inside a body should it be used in patients. Optical methods for in vivo imaging are all faced with the problem of limited transmission of light through tissues [[1], p. 237]. Because the human body absorbs and scatters photons in the visible and near infrared ranges with the mean-free-path in the sub-millimeter domain, such a source cannot be effectively detected on the body surface [4].

In this paper, we propose a computational optical biopsy (COB) method [5] to supplement and enhance the capabilities of fluorescent molecular tomography and bioluminescence tomographic, especially for their potential uses in patients. In order to detect the light source in a region of interest deep inside a subject, we can use a fiberoptical probe to detect the light source directly in the subject along one or multiple biopsy paths and next to compute the parameters or features of the embedded light source.

Several optical biopsy needle systems are already in operation [6-8], which have indicated the physical feasibility of this COB project. While similar to existing optical biopsy procedures in using fiber-optic probes [6-8], the proposed COB system and methods depend on not only optical devices but also advanced modeling and computation techniques to reconstruct an underlying source intensity distribution and extract its features of interest such as source center and effective intensity. There are several distinctions that substantiate our innovations. Our COB approach relies on sophisticated signal modeling and estimation from data collected along a number of biopsy paths, while other biopsy/endoscopic techniques perform direct anatomical imaging on speci c spots only. Our COB targets source intensity distributions triggered by probes instead of tissue/vascular properties that are concerned by other biopsy/endoscopic methods. Our СОВ intends to sense both fluorescent and bioluminescent sources, not just fluorescent sources as some optical endoscopic/spectroscopic techniques are designed for.

Mathematically we will Consider

$$
\left\{\begin{array}{r}
-\operatorname{div}\left(\mathrm{D} \nabla u_{0}\right)+\mu_{\alpha} u_{0}=f \text { in } \mathbb{R}^{3} \\
u_{0}(\infty)=0
\end{array}\right.
$$

and $\vec{t}=\left(t_{1}, t_{2}, t_{3}\right) \cdot v=\left(v_{1}, v_{2}, v_{3}\right)$ which has a fixed angle where $u_{0}$ is the average photon flux in all directions, $D(x)=\frac{1}{3\left(\mu_{\alpha}+\mu_{\mathrm{s}}^{\prime}\right)}, \mu_{\alpha}$ are positive constants with $\mu_{a}$ and $\mu_{s}$ being the absorption and scattering constants respectively in $\mathbb{R}^{3}$ and $f$ is either a measure or a $L^{\infty} \cap L^{1}$ function, which represents the light source.

\section{Single point source}

We will first consider the case where the source term $f=\sum_{i=1}^{N} \lambda_{i} \delta_{x^{i}}(\mathrm{x}) \quad$, where $\delta$ is the Dirac operator. In this case we have

$$
\begin{aligned}
& u_{0}(x)=\mathrm{C} \cdot \sum_{\mathrm{i}=1}^{\mathrm{N}} \frac{\lambda_{\mathrm{i}}}{\left|x-x^{i}\right| \exp \left(\mu_{\text {eff }}\left|x-x^{i}\right|\right)}, \\
& \mu_{e f f}=\sqrt{\frac{\mu_{\alpha}}{D}}
\end{aligned}
$$

where $C$ is a constant such that

$$
\lim _{\varepsilon \rightarrow 0^{+}} C \int_{[x]=\varepsilon}\left[-\frac{\partial}{\partial v}\left(\frac{D}{|x| \exp \left(\mu_{\mathrm{eff}}|x|\right)}\right)\right] d S_{x}=1,
$$

or

$$
\begin{aligned}
& C=\frac{1}{4 \pi D}, \quad(4) \\
& \text { i.e., } \\
& u_{0}(x)=\frac{1}{4 \pi D} \sum_{i=1}^{N} \frac{\lambda_{i}}{\left|x-x^{i}\right| \exp \left(\mu_{\mathrm{eff}}\left|x-x^{i}\right|\right)} .
\end{aligned}
$$

Assume that the needle insertion follows a straight line $l$ : $\vec{x}(s)=\overrightarrow{0}+s \vec{t}$, where $\vec{t}$ is the direction of the insertion and $\overrightarrow{0}$ one point along the insertion (Figure 1 ).

Let $v$ be a direction that needle tip detects light from. Then the measurement along the line $l$ is given by

$$
\begin{aligned}
m(s) & =-\left.D \cdot \frac{\partial u_{0}}{\partial v}\right|_{s} \\
& =-\left.D \cdot \nabla u_{0} \cdot v\right|_{\vec{x}=\overrightarrow{0}+s \vec{t}}
\end{aligned}
$$

$\theta$ with the needle direction $\vec{t}$, i.e., $\cos (\theta)=t_{1} v_{1}+t_{2} v_{2}+$ $t_{3} v_{3}$ 


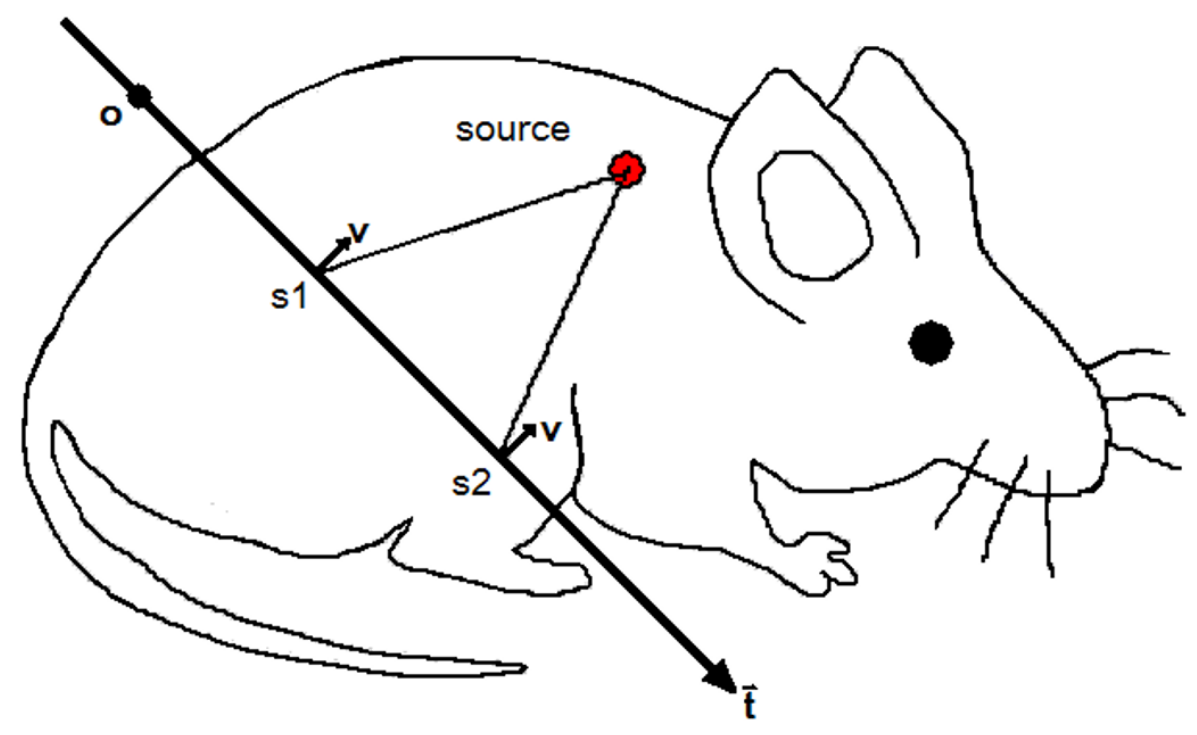

Figure I

A needle insertion path, where $t$ is the direction of the insertion, 0 one point along the insertion, $v$ a direction in which the needle tip detects light, $s_{1} ; s_{2}$ are points along the path

such that

$m(s)=\frac{1}{4 \pi} \sum_{i=1}^{N} \frac{\left(1+\mu_{\mathrm{eff}}\left|x-x^{i}\right|\right) \lambda_{i}}{\left|x-x^{i}\right|^{3} \exp \left(\mu_{\mathrm{eff}}\left|x-x^{i}\right|\right)}<x-x^{i}, v>$

Due to the notation and translation invariant properties of (1) and the design of the insertion needle, we may assume, without loss of generality, that

$$
\vec{x}(s)=s(1,0,0) \text { and } \theta=\frac{\pi}{2} .
$$

so that $v_{1}=0,\left(v_{2} v_{3}\right)=(\cos \alpha, \sin \alpha)$ and $x^{i}=\left(x_{1}^{i}, x_{2}^{i}, x_{3}^{i}\right)$. Then (7) becomes

$$
m(s)=\frac{1}{4 \pi} \sum_{i=1}^{N} \frac{\lambda_{i}\left(1+\mu_{\text {eff }} \sqrt{\left(s-x_{1}^{i}\right)^{2}+x_{2}^{i^{2}}+x_{3}^{i^{2}}}\right)\left(-x_{2}^{i} \cos \alpha-x_{3}^{i} \sin \alpha\right)}{\left[\left(s-x_{1}^{i}\right)^{2}+x_{2}^{i^{2}}+x_{3}^{x^{2}}\right]^{3 / 2} \exp \left(\mu_{\mathrm{eff}} \sqrt{\left(s-x_{1}^{i}\right)^{2}+x_{2}^{i^{2}}+x_{3}^{i^{2}}}\right)} .
$$

Now let $w^{i}=\sqrt{x_{2}^{i^{2}}+x_{3}^{i^{2}}}$.

Assuming that each insertion line can detect sources from two different angles $\alpha_{1}, \alpha_{2}$ such that $\alpha_{1}-\alpha_{2} \neq k \pi, k \in{ }_{ \pm} \mathbb{N}$, then

$$
\left[\begin{array}{cc}
\cos \alpha_{1} & \cos \alpha 2 \\
\sin \alpha_{1} & \sin \alpha_{2}
\end{array}\right]
$$

is non-singular. Therefore appropriate combination $\alpha_{1}$ and $\alpha_{2}$ depending on $\gamma$ of the measurements $m(s)$ would single out $x_{2}^{i}, x_{3}^{i}$, so that without loss of generality, we may assume that 
$\left\{\begin{array}{l}m_{k}(s)=\frac{1}{4 \pi} \sum_{i=}^{N} \frac{\lambda_{i} x_{k}^{i}\left[1+\mu_{\text {eff }} \sqrt{\left(s-x_{1}^{i}\right)^{2}+w^{i^{2}}}\right]}{\left[\left(s-x_{1}^{i}\right)^{2}+w^{i^{2}}\right]^{3 / 2} \cdot \exp \left(\mu_{\text {eff }} \sqrt{\left(s-x_{1}^{i}\right)^{2}+w^{i^{i^{2}}}}\right)} \\ k=2,3, s \in(-\infty, \infty),\end{array}\right.$

which are the measurements such that $\left\langle x-x^{i}, v>=x_{k}^{i}, k\right.$ $=2,3$ after combinations of $\alpha_{1}$ and $\alpha_{2}$ mentioned above and define

$m_{k}^{\ell} \equiv \int_{-\infty}^{\infty} m_{k}(s) s^{\ell} d s$, for integer $\ell=0,1,2, \cdots$

\section{Theorem 2.I}

(Single point source) If $N=1$, i.e.: if $u_{0}(x)=\frac{1}{4 \pi D} \frac{\lambda}{\left|x-x^{1}\right| \exp \left(\mu_{\mathrm{eff}}\left|x-x^{1}\right|\right)}$, then one insertion would uniquely determine $\lambda, x^{1}$, and $\mu_{\text {eff }}$ provided that the insertion line does not go through the point of the source. (See remark.)

\section{Proof}

In this case since

$m_{k}(s)=\frac{1}{4 \pi} \frac{\lambda x_{k}\left[1+\mu_{\mathrm{eff}} \sqrt{\left(s-x_{1}\right)^{2}+w^{2}}\right]}{\left[\left(s-x_{1}\right)^{2}+w^{2}\right]^{3 / 2} \exp \left(\mu_{\mathrm{eff}} \sqrt{\left(s-x_{1}\right)^{2}+w^{2}}\right)}, s \in(-\infty, \infty)$

$\bar{m}(s) \equiv m_{2}^{2}(s)+m_{3}^{2}(s)=\frac{1}{16 \pi^{2}} \frac{\lambda^{2} w^{2}\left[1+\mu_{\text {eff }} \sqrt{\left(s-x_{1}\right)^{2}+w^{2}}\right]^{2}}{\left[\left(s-x_{1}\right)^{2}+w^{2}\right]^{3} \exp \left(2 \mu_{\text {eff }} \sqrt{\left(s-x_{1}\right)^{2}+w^{2}}\right)}$.

Differentiate (13) with respect to $s$, we have

$$
\bar{m}^{\prime}(s)
$$

$$
=-\frac{\lambda^{2} w^{2}\left[1+\mu_{\text {eff }} \sqrt{\left(s-x_{1}\right)^{2}+w^{2}}\right]\left[\mu_{\text {eff }}^{2}\left(\left(x-x_{1}\right)^{2}+w^{2}\right)+3+3 \mu_{\text {eff }} \sqrt{\left(s-x_{1}\right)^{2}+w^{2}}\right]}{8 \pi^{2}\left[\left(s-x_{1}\right)^{2}+w^{2}\right]^{4} \exp \left(2 \mu_{\text {eff }} \sqrt{\left(s-x_{1}\right)^{2}+w^{2}}\right)} \cdot\left(s-x_{1}\right)
$$

such that the critical point of $\bar{m}(s)$ uniquely identifies $x_{1}$. Next with $x_{1}$ identified, we obtain

$$
\bar{m}\left(x_{1}\right)=\frac{\lambda^{2} w^{2}\left(1+\mu_{\mathrm{eff}} w\right)^{2}}{16 \pi^{2} w^{6} \cdot \exp \left(2 \mu_{\mathrm{eff}} w\right)}
$$

or

$$
\lambda^{2}=\frac{16 \pi^{2} w^{4} \exp \left(2 \mu_{\mathrm{eff}} w\right)}{\left(1+\mu_{\mathrm{eff}} w\right)^{2}} \bar{m}\left(x_{1}\right)
$$

And by taking the derivatives of $\bar{m}$ and evaluating them at $x_{1}$, we obtain that

$$
\begin{aligned}
& \frac{\bar{m}^{\prime \prime}\left(x_{1}\right)}{\bar{m}\left(x_{1}\right)} \equiv b=\frac{2\left(z^{2}+3 z+3\right)}{(z+1) w^{2}}, \quad(18) \\
& \frac{\bar{m}^{(4)}\left(x_{1}\right)}{\bar{m}\left(x_{1}\right)} \equiv a=\frac{6(z+2)\left(2 z^{3}+9 z^{2}+18 z+12\right)}{(z+1)^{2} w^{4}}
\end{aligned}
$$

where $z=\mu_{\mathrm{eff}} w$.

From (18) we obtain

$w=\sqrt{\frac{2\left(z^{2}+3 z+3\right)}{(z+1) b}}$

and plugging it into (19) we have

$\frac{2 a}{3 b^{2}}=\frac{(z+2)\left(2 z^{3}+9 z^{2}+18 z-12\right)}{\left(z^{2}+3 z+3\right)^{2}}=f(z)$.

If $f(z)$ is monotone in $z>0$, then (21) would give us a unique solution $z>0$.

We compute and obtain

$$
f^{\prime}(z)=-\frac{z\left(z^{3}+9 z^{2}+27 z+24\right)}{2\left(z^{2}+3 z+3\right)}<0,
$$

i.e., we have uniquely solved by the information from $\bar{m}$ the values of $\mu_{\mathrm{eff}}, w$ and therefore $\lambda$ by (17).

Next if we let $x_{2}=w \cos \beta, x_{3}=w \sin \beta$, then we get

$\tan \beta=\frac{m_{3}^{0}(s)}{m_{2}^{0}(s)}$

which could uniquely determine $\beta$ by the sign conditions of $m_{k}^{0}, k=2.3$.

That is, by now, we are able to determine, $\left(x_{1}, x_{2}, x_{3}\right), \mu_{\text {eff }}$ and $\lambda$; i.e. complete information about the source.

\section{Remark}

In case that the insertion line goes through the point of the source, which is verified by having each 
$m_{k}(s)=0, s \quad \mathbb{R} \backslash\{$ some single point $\}$

then only the two components of the point source, orthogonal to the insertion can be identified, which is the best possible. On the other hand, in practice, such events would have probability zero!

\section{Single ball source}

Next we consider ball sources, i.e., we assume that

$f=\sum_{i=1}^{N} \lambda_{i} \chi_{B\left(x^{i}, r^{i}\right)}(x)$,

where $\chi \Omega$ is the characteristic function of $\Omega$ and $B(y, r)=\left\{x \quad \mathbb{R}^{3}:|x-y|<r\right\}$.

For such source we have where

$$
\begin{aligned}
u_{0}(x) & =\sum_{i=1}^{N}\left\{\frac{\lambda_{i}}{\mu_{a}}\left[1-\frac{1+\mu_{e f f} r^{i}}{\exp \left(\mu_{e f f} r^{i}\right)} \cdot \frac{\sinh \left(\mu_{e f f}\left|x-x^{i}\right|\right)}{\mu_{e f f}\left|x-x^{i}\right|}\right] \chi_{B\left(x^{i}, r^{i}\right)}(x)\right. \\
& \left.+M\left(\lambda_{i}, r^{i}\right) \frac{1}{4 \pi D} \cdot \frac{1}{\left|x-x^{i}\right| \exp \left(\mu_{e f f}\left|x-x^{i}\right|\right)} \cdot\left[1-\chi_{B\left(x^{i}, r^{i}\right)}(x)\right]\right\},
\end{aligned}
$$

where

$M(\lambda, r) \equiv \int_{0}^{r} \lambda \tau^{2} \varphi(\tau) d \tau$ - the total intensity of the source in the form of $\lambda \chi_{B(o, r)}$.

Again we will discuss the single ball source first, i.e. $N=1$, so that we have

$$
\begin{aligned}
u_{0}(x) & =\frac{\lambda_{1}}{\mu_{a}}\left[1-\frac{1+\mu_{\mathrm{eff}} r^{1}}{\exp \left(\mu_{\mathrm{eff}} r^{1}\right)} \frac{\sinh \left(\mu_{\mathrm{eff}}\left|x-x^{1}\right|\right)}{\mu_{\mathrm{eff}}\left|x-x^{1}\right|}\right] \chi_{B\left(x^{1}, r^{1}\right)}(x) \\
& +M\left(\lambda_{1}, r^{1}\right) \frac{1}{4 \pi D} \cdot \frac{1}{\left|x-x^{1}\right| \exp \left(\mu_{\mathrm{eff}}\left|x-x^{1}\right|\right)}\left[1-\chi_{B\left(x^{1}, r^{1}\right)}(x)\right] .
\end{aligned}
$$

Theorem 3.I

(Single ball source) If $N=1$; i.e. if $u_{0}$ is given by (25), then one insertion would uniquely determine $M\left(\lambda_{3}, r^{1}\right), x^{1}$ and $\mu_{e f f i}$ in case the line stays outside of $B\left(x^{1}, r^{1}\right)$. In case the insertion line enters the interior of $B\left(x^{1}, r^{1}\right)$, then $x^{1}, r^{1}, \lambda_{1}$ and $\mu_{e f f}$ are uniquely determined by one insertion.

\section{Proof}

Case I

If the insertion line (refer to Theorem 1.1 in Section 2) stays outside $B\left(x^{1}, r^{1}\right)$, then the detected $u_{0}$ is given by

$$
u_{0}(x)=M\left(\lambda_{1}, r^{1}\right) \cdot \frac{1}{4 \pi D} \frac{1}{\left|x-x^{1}\right| \exp \left(\mu_{e f f}\left|x-x^{1}\right|\right)}
$$

i.e., $u_{0}(x)$ behaves exactly as a single point source with an intensity of $M\left(\lambda_{1}, r^{1}\right)$ and hence Theorem 2.1 guarantees the result.

Case 2

If the insertion line enters the support of the ball source $B$ $\left(x^{1}, r^{1}\right)$ but misses the center point $x^{1}$, then again due to the rotation and translation invariant properties of (1), we may assume, without loss of generality that the insertion is given by (8) and $x^{1}=\left(x_{1}, x_{2}, x_{3}\right)$ and hence the detection given by (6) is now

$m(s)$

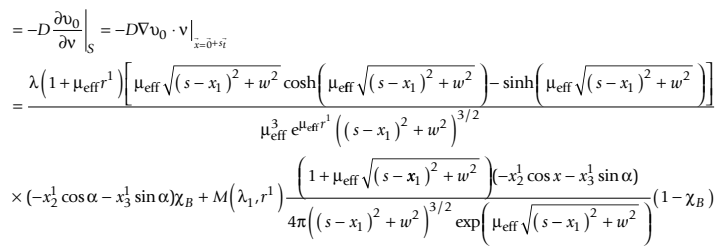

where $w=\sqrt{x_{2}^{2}+x_{3}^{2}}$.

Again, assuming that each insertion line can detect sources from two different angles $\alpha_{1}, \alpha_{2}$ such that $\alpha_{1}-\alpha_{2} \neq$

$k \pi, k \in \pm \mathbb{N}$, then

$\left[\begin{array}{cc}\cos \alpha_{1} & \cos \alpha 2 \\ \sin \alpha_{1} & \sin \alpha_{2}\end{array}\right]$

is non-singular. Therefore appropriate combination of the two measurements depending only on $\alpha_{1}$ and $\alpha_{2}$ would single out $x_{2}^{1}$ and $x_{3}^{1}$, so that without loss of generality we may assume that

$$
\begin{aligned}
& m_{k}(s)=x_{k}^{1} \phi(s), \text { where } \\
& =\frac{\lambda\left(1+\mu_{\text {eff }} r^{1}\right)\left[\mu_{\text {eff }} \sqrt{\left(s-x_{1}\right)^{2}+w^{2}} \cosh \left(\mu_{\text {eff }} \sqrt{\left(s-x_{1}\right)^{2}+w^{2}}\right)-\sinh \left(\mu_{\text {eff }} \sqrt{\left(s-x_{1}\right)^{2}+w^{2}}\right)\right]}{\mu_{\text {eff }}^{3} \mathrm{e}^{\mu_{\text {eff }} r^{1}}\left(\left(s-x_{1}\right)^{2}+w^{2}\right)^{3 / 2}} \\
& \quad \times \chi_{B}+M\left(\lambda_{1}, r^{1}\right) \frac{1+\left(\mu_{\text {eff }} \sqrt{\left(s-x_{1}\right)^{2}+w^{2}}\right)}{4 \pi\left(\left(s-x_{1}\right)^{2}+w^{2}\right)^{3 / 2} \exp \left(\mu_{\text {eff }} \sqrt{\left(s-x_{1}\right)^{2}+w^{2}}\right.}\left(1-\chi_{B}\right) .
\end{aligned}
$$

If we let $x_{2}=w \cos \beta, w_{3}=w \sin \beta$, then

$$
\tan \beta=\frac{m_{3}^{0}(s)}{m_{2}^{0}(s)},
$$

which would uniquely determine $\beta$ by the above question and the sign conditions if $M_{k}^{0}, k=2.3$.

Define 
$\bar{m}(s)=m_{2}(s)^{2}+m_{3}(s)^{2}=w^{2} \phi^{2}(s)$.

Note that since in this case we assume that the insertion line enters the interior of $B\left(x^{1}, r^{1}\right)$ but misses the center $x^{1}$, we have $0<w<r^{1}$ and the line would enter and leave $B$ $\left(x^{1}, r^{1}\right)$ when

$$
\left\{\begin{array}{c}
\sqrt{\left(s-x_{1}\right)^{2}+w^{2}}=r^{1} \text { or } \\
s=x_{1} \pm \sqrt{r^{1^{2}}-w^{2}}
\end{array}\right.
$$

which is exactly where our measurement $M_{k}(s)$, or equivalently $\phi(s)$ fails to be differentiable. Therefore let $s_{\text {in }}$ and $s_{\text {out }}$ be two points where we observe the jump discontinuity of $\bar{m}^{1}(s)$.

Then we have

$$
\left\{\begin{array}{c}
x_{1}=\frac{s_{\text {in }}+s_{\text {out }}}{2} \\
r^{1^{2}}=w^{2}+\left(\frac{s_{\text {out }}-s_{\text {in }}}{2}\right)^{2}
\end{array}\right.
$$

(32) together with (29) uniquely identify $x_{1}$ and establish a relation between $r_{1}$ and $w$. Then we are able to evaluate $\bar{m}\left(x_{1}\right)$ to get

$$
\begin{aligned}
\bar{m}\left(x_{1}\right) & =w^{2} \cdot \varphi^{2}\left(x^{1}\right) \\
& =\frac{w^{2} \lambda_{1}^{2}\left(1+\mu_{\mathrm{eff}} r^{1}\right)^{2}\left[\mu_{\mathrm{eff}} w \cosh \left(\mu_{\mathrm{eff}} w\right)-\sinh \left(\mu_{\mathrm{eff}} w\right)\right]^{2}}{\mu_{\mathrm{eff}}^{6} \exp \left(2 \mu_{\mathrm{eff}} r^{1}\right) w^{6}},
\end{aligned}
$$

i.e.,

$$
\lambda_{1}^{2}=\frac{\exp \left(2 \mu_{e f f} r^{1}\right) \cdot \omega^{4} \cdot \mu_{\text {eff }}^{6}}{\left(1+\mu_{e f f} r^{1}\right)^{2}\left[\mu_{e f f} \omega \cosh \left(\mu_{e f f} \omega\right)-\sinh \left(\mu_{e f f} \omega\right)\right]^{2}} \bar{m}\left(x_{1}\right) .
$$

Next by taking the derivatives of $\bar{m}$ and evaluating them at $x_{1}$, we obtain that

$$
\begin{aligned}
& \frac{\bar{m}^{\prime \prime}\left(x_{1}\right)}{\bar{m}\left(x_{1}\right)} \equiv b=\frac{3 \sinh (z)-3 z \cosh (z)+z^{2} \sinh (z)}{\omega(z \cosh (z)+z \cosh (z)) \cdot} \\
& \frac{\bar{m}^{(4)}\left(x_{1}\right)}{\bar{m}\left(x_{1}\right)} \equiv a=\frac{3\left[-15 \sinh (z)+15 z \cosh (z)-6 z^{2} \sinh (z)+z^{3} \cosh (z)\right]}{w(-\sinh (z)+z \cosh (z)) \cdot}
\end{aligned}
$$

>From (35) we obtain $w=\sqrt{\frac{3 \sinh (z)-3 z \cosh (z)+z^{2} \sinh (z)}{b(-\sinh (z)+z \cosh (z))}}$

and plugging it into (36) we get

$$
\begin{aligned}
\frac{a}{3 b^{2}} & =\frac{[-\sinh (z)+z \cosh (z)]\left[-15 \sinh (z)+15 z \cosh (z)-6 z^{2} \sinh (z)+z^{3} \cosh (z)\right]}{\left[3 \sinh (z)-3 z \cosh (z)+z^{2} \sinh (z)\right]^{2}} \\
& =f(z) .
\end{aligned}
$$

which can be veri ed to be an increasing function. Therefore (38) has a unique solution $z$ and (37) then uniquely de nes the $w$ which in turn defines $\lambda_{1}$ uniquely by (34) and $r^{1}$ uniquely by (32).

\section{Discussions and conclusion}

We have demonstrated the modeling of computational optical biopsy with the diffusion optics, the solution uniqueness properties in two typical configurations and provide explicit formulas for the reconstruction of both optical properties and source parameters. Mathematically, one single insertion will be enough to estimate the above parameters. However, physically, more measurements will guarantees the robustness of the estimates. The needle trajectory can also be dynamically restarted and optimized towards the center of the source based on the successive estimate. Further investigation on multiple point sources and multiple ball sources is undergoing. For example the double sources probelm can be handled in such a way that by using the moments defined (11) one could reduce the problem to a equivelent one with a few freedom and thus a numerical optimization technique will be used to solve the inverse problems.

\section{Contributions}

The three authors made about equal contributions in this work.

\section{Acknowledgements}

This work is partially supported by the National Institutes of Health (EB00I685 and EB002667).

\section{References}

I. Contag $\mathrm{CH}$, Bachmann $\mathrm{MH}$ : Advances in vivo bioluminescence imaging of gene expression. Annual Review Of Biomedical Engineering 2002, 4:235-260.

2. Wang G, et al.: Development of the first bioluminescent tomography system. Radiology Suppl. (Proceedings of the RSNA) 2003.

3. Wang G, Li Y, Jiang M: Uniqueness theorems for bioluminescent tomography. Medical Physics 2004, 3 I (8):2289-2299.

4. Beuthan J, Mahnke C, Netz U, Minet O, MÃller G: Optical molecular imaging: Overview and technological aspects. Medical Laser Application 2002, I 7(I):25-30.

5. Wang G, Li Y, Jiang M: Computational optical biopsy methods, techniques and apparatus. Provisional patent application filed in March 2005 (Patent disclosure filed with Univ. of lowa Research Foundation in December 2003).

6. Liu Q, Ramanujam N: Experimental proof of the feasibility of using an angled fiber-optic probe for depth-sensitive fluorescence spectroscopy of turbid media. Optics Letters 2004, 29(I 7):2034-2036. 
7. Li XD, Chudoba C, et al:: Imaging needle for optical coherence tomography. Optics Letters 2000, 25(20):1520-1522.

8. Alfano RR, Katz A: Optical biopsy V: 27-28 January San Jose, USA. Progress in biomedical optics and imaging; v. 5, no. 15. 2004, Bellingham, Wash.: SPIE. ix :I34.

Publish with Bio Med Central and every scientist can read your work free of charge

"BioMed Central will be the most significant development for disseminating the results of biomedical research in our lifetime. " Sir Paul Nurse, Cancer Research UK

Your research papers will be:

- available free of charge to the entire biomedical community

- peer reviewed and published immediately upon acceptance

- cited in PubMed and archived on PubMed Central

- yours - you keep the copyright

Submit your manuscript here:

http://www.biomedcentral.com/info/publishing_adv.asp 